\title{
Progress in Traction Battery Recycling Industry for Electric Vehicles
}

\author{
Ying-hao $\mathrm{XIE}^{1,2,3,{ }^{*}}$, Hai-jun $\mathrm{YU}^{1,2,4}$, Yan-nan $\mathrm{OU}^{1,3}$ and Chang-dong $\mathrm{LI}^{2,3}$ \\ ${ }^{1}$ Guangdong Brunp Recycling Technology Co., Ltd., Foshan 528244, China \\ ${ }^{2}$ Hunan Brunp EV Recycling Co., Ltd, Changsha 410600, China \\ ${ }^{3}$ Guangdong Traction Battery \& Electric Vehicle Recycling Research Academician Workstation, \\ Foshan 528244, China \\ ${ }^{4}$ Graduate School of Central South University, Changsha 410083, China \\ *Corresponding author: diangong306@126.com
}

Keywords: EV battery, Recycling processing, industry, electric vehicles.

\begin{abstract}
With the strong support of the government, China's new energy automotive industry has developed rapidly, and the quantity of the waste EV batteries have been produced. From its huge quantity, environmental protection and resource regeneration point of view, waste lithium-ion batteries have a high recovery value and potential. This paper summarizes the current policy requirements of the Chinese government on the recycling of $\mathrm{EV}$ batteries and the development of related enterprises in the industry, focusing on the progress of physical recovery and chemical recovery of EV battery recycling. The existing problems are how to implement the policy, how to recover low-value materials, explore the power of the industry chain circulation. The future development trend is to study the sustainable development of the recovery model and industry chain loopholes in the industry common problems.
\end{abstract}

\section{Introduction}

In 2012, the State Council issued the "Energy and New Energy Automotive Industry Development Plan" (Guo Fa [2012] No. 22). China's new energy automotive industry has been officially recognized by China's policy, the new energy automotive industry began to force. After several years of development, according to the Ministry of Industry released data, in 2014 the new energy vehicle production 78,000, 2015 explosive growth of 3 times, reaching 340,000, 2016 to maintain steady growth, in 2016 reached 517,000.

With the new energy automotive industry is gradually mature, the new energy industry backend market - EV battery recycling industry has gradually been the government attention. The Chinese government has introduced a series of policy documents to make a request for EV battery recycling.

January 2016, the Development and Reform Commission led the release of the "Electric vehicle EV battery recycling technology policy (2015 version)", and clear the four main responsibility for recycling EV battery (automobile manufacturers, battery manufacturers, echelon use of enterprises, scrap car recycling enterprises ). But also clear the recycling business, battery sales, demolition requirements, storage requirements, transport requirements, discharge requirements, dismantling requirements, pyrolysis requirements, broken sorting requirements, smelting requirements.

February 2016, the Ministry of Industry issued a "new energy vehicle waste EV battery comprehensive utilization industry standard conditions" and "new energy vehicles waste EV battery comprehensive utilization industry standard notice management Interim Measures", clear the recycling enterprises should have the size, equipment, Technology, comprehensive utilization of resources, energy consumption, environmental protection requirements, product quality, vocational education, safety production and social responsibility requirements.

December 2016, the Ministry of Industry on the "new energy vehicle EV battery recycling Interim Measures" (draft) asked for public comments. The "new energy vehicle EV battery recycling Interim Measures" (draft) provides the requirements of the design stage, production stage, recovery stage, sales stage, use and maintenance stage, and scrapped stage. Also provides the user's responsibility 
requirements, clear the collection, storage, transportation, comprehensive utilization principles, the overall requirements of enterprises, the use of cascade requirements, cascade use of battery requirements, and recycling requirements. At the same time clear the establishment of the standard system, information filing system, traceability management system, cascade use product management, incentive policies, management support, and social supervision. Finally, clear the penalties on the car companies, battery companies, and comprehensive utilization corporate.

\section{Industry Development}

As the development of new energy vehicles is too fast, the problem of backend processing has not yet attracted people's attention. China's EV battery recycling industry started late but develops fast. In line with policies and regulations of the EV battery recycling business is not much, and relatively concentrated business resources. Throughout the world, the Chinese government to promote new energy vehicles, the largest, and China's EV battery recycling technology is relatively leading. In 2006, in other countries are still studying how to dismantle the EV battery, China has developed the first generation of mechanized EV battery dismantling equipment [1]; In 2011, in other countries to study the mechanized dismantling equipment, China has developed a fully automated battery dismantling equipment [2]. China's EV battery industry for the key elements of the recovery rate has been as high as $98 \%$, and as an important indicator into the national standard [3,4]. At present, China has four large-scale representative enterprises and the situation in table 1.

Table 1 China's four large-scale EV battery recycling enterprises

\begin{tabular}{|c|c|c|c|}
\hline Company name & Processing scale (tons / year) & Processing technology & Number of patents / items \\
\hline Brunp & 100000 & Fire wet method joint & 135 \\
\hline GEM & 15400 & Fire wet method joint & 66 \\
\hline CY & 4000 & Fire wet method joint & 25 \\
\hline HP & 980 & Fire wet method joint & 45 \\
\hline
\end{tabular}

China has four large-scale EV battery recycling enterprises, and the design and processing capacity is not low, but the actual amount of recycling is far from the expected amount of scrap, and the design capacity is not reached. Although the Chinese government has introduced the relevant policies to require the EV battery specification recovery and processing, but the implementation is very difficult, the lack of mandatory execution, the policy is mostly to stay paper, not implemented, the market chaos, most of the battery flow to illegal processing channels.

\section{EV Battery Recycling Technical Exploration}

EV battery recycling process shows in Figure 1.

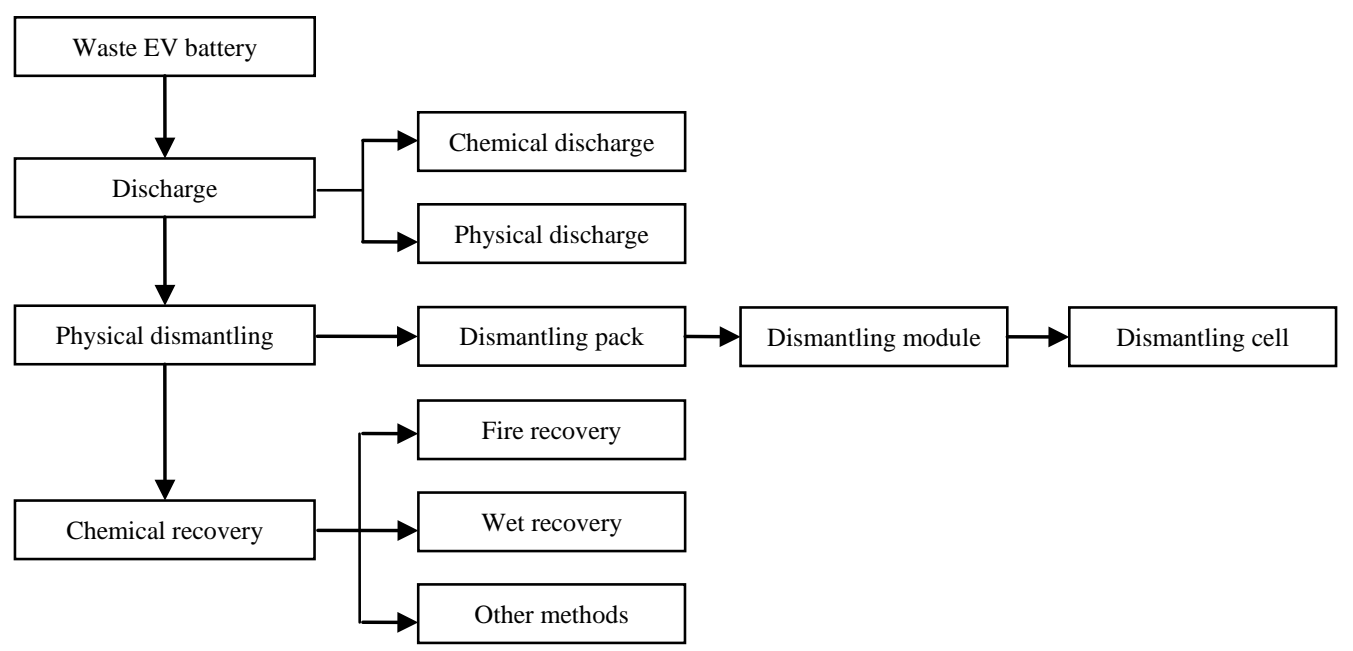

Figure 1 EV battery recycling flow chart 
Discharge. Physical dismantling includes three levels, respectively, for the battery pack dismantling, battery module dismantling, battery monomer dismantling. In order to ensure the safety of disassembly, it is usually necessary to discharge the battery. The discharge object may be a battery pack, a battery module, and a battery cell. Discharge methods are physical discharge and chemical discharge.

Physical Discharge. The use of physical discharge, requiring good performance of the battery pack, BMS and relay operation is normal. Discharge need to use a dedicated communication tool, the conventional physical discharge battery voltage prone to rebound, the battery still has a high voltage, but most of the battery energy has been released, the risk is less than the discharge battery, but still has a certain risk Sex. Through a specific physical discharge method can be completely released from the battery energy, damage the battery internal active material, the battery voltage no longer rebound, but the need for special discharge equipment and technology.

Chemical Discharge. Chemical discharge on the battery performance is not required, whether it is good or damaged appearance, deformation, leakage of the battery monomer, module or battery pack can be used. Chemical discharge will completely damage the battery, resulting in the battery internal electrolyte, electrode active materials and other substances leaked out of the discharge solution. After discharge, the solution contains a lot of heavy metals and organic solvent and becomes very difficult to treatment. Therefore, in the general do not have the professional sewage treatment facilities and equipment of the vehicle business, $4 \mathrm{~S}$ shop, power storage temporary network and other places can not apply chemical discharge method. And the application of a wide range of small investment, low cost, technical difficulty is small, easy to batch processing and other advantages, chemical discharge is generally used in professional battery recycling enterprises [5].

Physical Recovery. Dismantling Pack. Passenger car space is limited, in the design and manufacture of EV battery pack is based on the structure of the body design, different manufacturers of different models of EV battery pack shape irregular, different shapes [6]. Commercial vehicles have a larger space, commercial vehicles have a certain mileage requirements, the use of more EV batteries, commercial vehicle EV battery is mainly considered the price and cost, the design is not too much on the external requirements, commercial vehicle battery pack Shape regular, shape is usually rectangular. For the shape of the car is not regular use of EV batteries, dismantling is considered more artificial dismantling. Shape of the commercial vehicle with a regular battery dismantling the future can be developed to the direction of automation. Dismantling the battery pack shell removed, and then remove the fixed module components, and then remove the high and low voltage wiring harness, the use of special mold out of the module, remove the cooling system.

Dismantling Module. Battery module can be divided into three types according to the structure: (1) hard shell monomer module; (2) soft package monomer module; (3) cylindrical monomer module.

Hard Shell Monomer Type Module. The module assembled by the hard shell monomer. Assembly methods are usually bolted and fixed. Disassemble the module shell directly cut off, and then remove the internal monomer. In order to improve the safety of dismantling, removal should be used insulation cutting tools, and attention to remove the debris generated to avoid short circuit. Removal should also pay attention to not damage the internal monomer. In the event of damage, internal electrolyte leakage, short circuit within the battery, prone to fire.

Flexible Package Type Module. The flexible package module is usually formed by connecting a plurality of single series strings in parallel to form a small module, and a plurality of small modules are arranged in series to form a large module group. The large module group is finally assembled into a battery pack. In the removal of large modules because the large module is usually a bolt connection or hoop lock, so the dismantling are relatively simple. But after the dismantling of large modules get small modules, small modules have protective cover protection is not easy to dismantle, the protective cover is usually a deformation of the thin steel plate, so easy to dismantle the design for the dismantling is very important Design should not be used. In the dismantling of small modules, the soft package between each other in order to avoid displacement, between the two is the use of adhesive cement, resulting in monomer and monomer separation becomes very difficult. If the soft 
package monomer can not directly into the broken sorting line, it will greatly affect the recovery efficiency.

Cylindrical Monomer Module. The cylindrical monomer module is fixed in a plastic frame by a large number of cylindrical monomers. If the cylinder module is dismantled, it is time consuming and labor intensive. The cost is high, and the cylindrical monomer can be used in the recovery Can directly enter the broken line of the characteristics of direct access to the production line, directly skip the module dismantling the step

Dismantling Cell.The battery can be divided into three categories according to the dismantling method, 1 , hard shell square single battery; 2 , soft package single battery; 3 , cylindrical single cell.

Hard Shell Square Cell. Hard shell square cell from the aluminum alloy or iron metal as a shell, if the direct feed into the pyrolysis and crushing production line, it will introduce a lot of iron impurities, increasing the cost of the late recovery of valuable metals and cost. Therefore, it is necessary to physically separate the shell and the batteries before entering the chemical recovery.

Hard shell square battery can be dismantled when the end of the battery ear can be cut, after cutting the use of reverse push or gripping the way the monomer within the battery removed. This disassembly operation is easy to implement, but because of the oppression of the sidewall of the monomer, the cell is not easily removed, and the batteries remaining inside the monomer directly reduce the recovery of the valuable metal. Or the side of the monomer side of the largest side of the edge along the line cutting, cut out after the removal of batteries. It is also possible to cut a circle around the other four sides of the monomer along the opposite side of the non-side area [7]. Behind the two dismantling methods easy to achieve automation, and can be efficient recovery of batteries.

Soft Package Cell. Soft package single battery shell material for the aluminum plastic film, aluminum plastic film in the pyrolysis stage can be pyrolysis stage, aluminum foil can be with the positive film on the aluminum foil processing direction similar to broken after sorting out. Therefore, the soft package without the need for dismantling, processing can be directly into the broken sorting recovery line.

Cylindrical Cells. Cylindrical single cell market commonly used models are 18650, 22650, 26650, 32650, etc., generally smaller size, the huge number of battery pack, if each of its battery mechanized dismantling time-consuming energy consumption, and will reduce the production effectiveness. In the actual industrial recycling can be directly into the pyrolysis crushing production line.

Chemical Recovery. Fire Recovery. Fire recovery is mainly for waste nickel-metal hydride batteries to recover Ni-Fe alloy-based, the use of waste batteries in the boiling point of the difference between the elements of separation, melting. The specific steps are: first waste Ni-MH battery broken, disintegration, washing, to remove the $\mathrm{KOH}$ electrolyte. Gravity sort out the waste, and then into the roasting furnace for roasting. From the excretion of flue gas residue separation, purification of different metals. Ni-Fe alloy with 50\% -55\% nickel 30\% to 35\% iron can be obtained.

Fire recycling waste batteries usually do not appear alone, usually followed by a period of wet recovery process will be prepared into the final chemical products. After the alloy is recovered by the fire method, a wet step such as further processing, disintegration, dissolution, separation and purification of the alloy is required to form the compound or metal to be prepared. As the low boiling point of lithium, wet recovery of lithium-ion battery will make lithium volatile, lithium is not only easy to recover, but also become waste gas pollution caused by the environment.

Wet Recovery. Wet recovery is not required for high temperature roasting in relation to the fire, and no alloy is obtained. Hydrometallurgy is the battery pretreatment, the inorganic acid solution will waste batteries in the metal ions leaching, the metal ions into the solution, and then by precipitation, extraction, salting out, ion exchange, electrochemical and other methods to further separation , Nickel, cobalt, manganese, lithium and other metal elements, or the above leaching solution directly synthetic nickel cobalt aluminum hydroxide or nickel cobalt cobalt manganate and other cathode materials. Wet recovery process is relatively complex, the process is relatively long, but the recycling process is more sophisticated, high purity of recycled products, recyclable metal species, and the metal recovery rate is high. Most of China's industrial recycling of used batteries in this method. 
Other Recycling Methods. Some scholars have proposed the freezing method, biological method, flotation method, grinding method, organic solvent method, precipitation method, extraction method, salting-out method, electrochemical method and so on are not independent recovery method, but the recovery method used inside one process [8]. Such as the freezing method is a pretreatment method, the battery placed in a frozen environment for disassembly broken, the battery material can be reduced activity, to avoid fire and other phenomena, to achieve safe dismantling. Due to the high cost of manufacturing refrigeration environments, industrial applications are generally replaced by chemical discharges. Biological law is dissolved in the process of using special bacteria produced by the acid to dissolve the powder, due to long time to respond, the survival of bacteria more stringent conditions, the general application of less. Flotation method is through the flotation of the positive and negative material separation, grinding method is the use of solid-phase reaction of the cathode material into other materials and then dissolved and other recovery. Organic solvent method is the use of organic solvents to dissolve the binder so that the cathode material and aluminum foil from, and then to achieve recovery, low energy consumption, but the method will cause a certain degree of damage to material properties. The precipitation method, extraction method, salting-out method, electrochemical method belong to the wet recovery category, but using different means to purify the solution or metal ion separation method.

\section{Conclusion}

China's EV battery recycling technology on the development of the world's leading global, physical recovery and chemical recycling technology is mature, but the industry is in a relatively chaotic stage of development, recovery and measurement of waste is far from match, a lot of waste batteries into illegal channels. This is not a lack of policy requirements, the threat of EV battery awareness, strange new things and the lack of policy penalties lead to the development of China's EV battery recycling market confusion, the future development of the focus in addition to enhance the recovery of valuable metals, dismantling efficiency, Recovery costs, EV battery life cycle of the research can be seen outside the subject, but also should pay attention to the recovery model research, industry sustainable development research and the closure of the policy loopholes and other EV battery recycling common research topics.

\section{Acknowledgement}

This research was financially supported by the national Torch Program projects (No. 2013GH061426), National Key Technology R\&D Program alternative project (subproject) (No. 2014BAC03B01) and strategic emerging industries core technology research projects in Guangdong Province (No. 2011A032302001).

\section{References}

[1] C.D. Li, Recycle battery automatic dismantling machine, C.N. Patent, 200620059829.X.(2006).

[2] C.D. Li, X.D. Chen, J.J. Tan, et al. A waste battery water jet cutting machine, C.N. Patent, 201120305816.7. (2011).

[3] GB/T 33059-2016, Methods for disposal and recycling of lithium ion battery material wastes.(2016).

[4] HG/T 5019-2016, Recovery of nickel and cobalt in waste batteries.(2016).

[5] Y.H. Xie, HJ. Yu, Y.N. Ou and C.D. Li, Environmental impact assessment of recycling waste traction battery, Inorganic Chemicals Industry, 47(2015) 43-46, 61.

[6] GB/T 33598-2017, Recycling of traction battery used in electric vehicle-Dismantling specification.(2017). 
[7] HJ. Yu, Z. Chen and Y.H. Xie, Experimental research on harmful gas control of automated traction battery cell disassembling line, Industrial Safety and Environmental Protection, 42(2016) 39-41.

[8]Y.H. Xie, HJ. Yu, Y.N. Ou and C.D. Li, Electric vehicle identity recognition and traction battery coding, Chinese Journal of Power Sources, 40(2016) 113-116. 